\title{
Automated clean-up, separation and detection of polycyclic aromatic hydrocarbons in particulate matter extracts using a 2D-LC/2D-GC system: a method translation from two FIDs to two MS detectors
}

\author{
Hwanmi Lim $^{1} \cdot$ Trifa M. Ahmed ${ }^{1,2} \cdot$ Christoffer Bergvall $^{1,3} \cdot$ Roger Westerholm $^{1}$
}

Received: 7 June 2017 / Accepted: 4 July 2017 / Published online: 24 July 2017

(C) The Author(s) 2017. This article is an open access publication

\begin{abstract}
An online two-dimensional (2D) liquid chromatography/2D gas chromatography system with two mass-selective detectors has been developed on the basis of a previous system with two flame ionization detectors. The method translation involved the change of carrier gas from hydrogen to helium, column dimension and detectors. The 2D system with two mass-selective detectors was validated with use of polycyclic aromatic hydrocarbon (PAH) standards and two standard reference materials from air and diesel exhaust. Furthermore, the system was applied to a real sample, wood smoke particulates. The PAH values determined correlated well with the previous data and those from the National Institute of Standards and Technology. The system enhanced the benefits of the previous system, which were limited by the low detectability and lack of mass selectivity. This study shows an automated 2D system that is valid for PAH analysis of complex environmental samples directly from crude extracts.
\end{abstract}

Keywords Polycyclic aromatic hydrocarbon .

Multidimensional gas chromatography $\cdot$ Standard reference material · Wood smoke particulates $\cdot$ Long-term stability

Electronic supplementary material The online version of this article (doi:10.1007/s00216-017-0509-1) contains supplementary material, which is available to authorized users.

Roger Westerholm

roger.westerholm@aces.su.se

1 Department of Environmental Science and Analytical Chemistry (ACES), Stockholm University, 10691 Stockholm, Sweden

2 Livsmedelsverket, Box 622, 75126 Uppsala, Sweden

3 Department of Ecology and Genetics, Limnology, Uppsala University, 75236 Uppsala, Sweden

\section{Introduction}

Multidimensional chromatography (MDC) has been an alternative to single chromatography for analysis of environmental samples which may contain various compounds of interest as well as complex matrices [1]. Especially, multidimensional gas chromatography (MDGC) has had the main role because of its high separation capacity with the help of capillary gas chromatography (GC) columns [2]. A classic MDGC approach used so-called heart-cut via a flow switching device (GC-GC) located between the two columns to direct a fraction of the compounds from the first column to the second for further separation [3]. Another mode in MDGC is comprehensive $\mathrm{GC} \times \mathrm{GC}$, which was introduced later, where a modulator continuously collects and transfers small effluents from the first column to the second column [4]. Both modes are complementary and intended to maximize the peak capacity [5]. $\mathrm{GC}-\mathrm{GC}$ is more applicable for identifying and studying known compounds, whereas the latter mode is used more extensively for characterization and classification of known and unknown compounds $[6,7]$.

Polycyclic aromatic hydrocarbons (PAHs), a subgroup of polycyclic aromatic compounds [8], are ubiquitous organic compounds found in the environment. PAHs are formed during incomplete combustion of organic matter mainly from anthropogenic activities (e.g. fossil fuel combustion, biomass burning, cooking and tobacco smoking) [9]. They are known to be carcinogenic and reprotoxic and for causing cardiovascular disease in animal studies $[9,10]$. The International Agency for Research on Cancer classified benzo $[a]$ pyrene as carcinogenic to humans, being the only compound in group 1 among the PAHs $[11,12]$. The International Agency for Research on Cancer recently added outdoor air pollution to group 1, reinforcing the health risk of PAHs as they constitute the outdoor air with 
other possible sources, including ozone, particulate matter, sulfur dioxide and carbon monoxide [13].

The environmental and health-perspective importance of PAHs has driven the development of analytical methods capable of separating a range of compounds from low to high molecular weight as well as isomers and alkylated homologues from different matrices [14, 15]. MDC-based PAH analysis was one of the choices as it could simplify the complexity of the samples. A liquid chromatography (LC)-based MDC method was developed to maximize the separation of the historical 16 PAHs listed by the US Environmental Protection Agency [16]. In detail, a fractionation based on normal-phase LC first separated the PAHs according to the number of aromatic rings, then the following reversed-phase LC separated each fraction, which was later developed into an online system [17]. The multidimensional LC (MDLC) technique, however, was limited in use to certification of standard reference materials (SRMs) and could not be used for routine analysis [15]. Another MDC approach using GC expanded the number of analytes with increased peak capacity [15]. Heartcut $\mathrm{GC}-\mathrm{GC}$, more conventional MDGC, is suitable for medium to complex samples with 100-200 analytes, and a comparably novel technique, comprehensive $\mathrm{GC} \times \mathrm{GC}$, fits well with samples with high complexity [6]. The selection of appropriate column phases plays a key role in the multidimensional chromatographic separation [15, 18-20]. Amino and polymeric $\mathrm{C}_{18}$ LC columns, for instance, became preferable for MDLC, with the first column used for clean-up and isolation of the PAH fraction of interest from complex samples and the second column used for selective separation of each fraction containing PAHs with analytical difficulties (e.g. isomers or alkyl substituents) [21]. In MDGC, various GC columns have also been used to improve the separation mainly with capillary columns; for example, 5\% and 50\% phenyl methylpolysiloxane phases and/or 50\% liquid crystal polysiloxane (LC50) $[14,15]$. Columns with a $50 \%$ phenyl phase or similar especially demonstrated improved separation for isomeric PAHs of chrysene/triphenylene and benzo[b]fluoranthene/benzo[j]fluoranthene than a 5\% phenyl phase, being the column of choice in PAH analysis [15]. An LC50 column, on the other hand, has been used as the other dimension in MDGC because of its shape selectivity which is similar to that of polymeric $\mathrm{C}_{18}$ phases, especially useful for isomer separation [22-24]. The 50\% phenyl and LC50 columns were used in the previous two-dimensional (2D) GC system as the first and second dimension respectively [25]. Meanwhile, a novel phase called a nano stationary phase was introduced in comprehensive $\mathrm{GC} \times \mathrm{GC}$ as the second dimension to maximize the chromatographic orthogonality [23, 24].

A 2D system combining MDLC and MDGC was developed in which 2D-LC performed the sample clean-up and the fractionation enriched with PAHs, followed by 2D-GC analysis using heart-cut GC-GC [25]. The system was also shown to be valid for analysis of PAHs from urban dust and diesel engine exhaust particulates [25]. The detector used, a flame ionization detector (FID), however, limited the benefits of the system because of low detectability and lack of mass selectivity. The present study mainly aimed at method translation, changing the detector from an FID to a mass-selective detector (MSD), consequently entailing the use of helium instead of hydrogen as the carrier gas. The 2D system with an MSD at each end of the GC columns was validated and further applied to three different environmental matrices from air, diesel and wood smoke particulates.

\section{Materials and methods}

\section{Chemicals and solvents}

All solvents (high-performance LC grade), including methanol, methyl tert-butyl ether (MTBE) and toluene, were purchased from Rathburn Chemicals (Walkerburn, UK). Anhydrous dodecane (purity 99\% or greater) was obtained from SigmaAldrich (St Louis, MO, USA). Dibenzothiophene (99\%) was purchased from Janssen Chimica (Beerse, Belgium). All the other PAHs, including deuterated internal standards (ISs), used in this study were described previously $[25,26]$. A full list of the PAHs and the ISs is presented in the electronic supplementary material, including the name, CAS Registry Number, and abbreviation (Table S1).

\section{Particulate samples}

The same SRMs, SRM 1649a (urban dust) and SRM 1975 (diesel particulate extract) from the National Institute of Standards and Technology (NIST; Gaithersburg, MD, USA) were used as in a previous study [25]. Additionally, wood smoke particulates were collected from combustion of birch and fir wood in a small cast-iron stove situated in an exposure chamber [27].

\section{Sample preparation}

SRM 1649a and SRM 1975 were prepared as described previously [25]. Briefly, $167 \mathrm{mg}$ of SRM 1649a and one ampoule of SRM 1975 were placed on glass fibre filters $(\mathrm{GF} / \mathrm{C} 47 \mathrm{~mm}$, Whatman, Maidstone, UK) and spiked with the ISs separately. Then, the filter was inserted into a $5-\mathrm{mL}$ extraction cell for pressurized liquid extraction (ASE 200 accelerated solvent extraction system, Dionex, Sunnyvale, CA, USA) using 9:1 $(\mathrm{v} / \mathrm{v})$ toluene/methanol as the extraction solvent at $20.7 \mathrm{MPa}$ and $200{ }^{\circ} \mathrm{C}$ for $30 \mathrm{~min}$ and with five static cycles [28]. A blank filter was prepared in the same manner. Additionally, $3.6 \mathrm{mg}$ of wood smoke particulates was weighed and prepared as well. Approximately, $90 \%$ of the whole extract was used for 
the previous study using 2D-LC/2D-GC/flame ionization detection [25], and the remaining amount was used for the LCGC/mass spectrometry (MS) analysis and the present study. All the extracts used for the present study were stored in a freezer at $-18{ }^{\circ} \mathrm{C}$ until analysis. The storage period of the crude extracts was nearly 4 years.

\section{Instrumentation}

All the instrumentation for the 2D-LC/2D-GC system was described in the previous study [25]. The 2D-LC system was composed of three LC columns: $\mathrm{C}_{18}, 250 \mathrm{~mm} \times 4.6 \mathrm{~mm}, 5 \mu \mathrm{m}$ (Phenomenex, Torrance, CA, USA); Cosmosil 5pentabromobenzyloxypropyl, $150 \mathrm{~mm} \times 4.6 \mathrm{~mm}, 5 \mu \mathrm{m}$ (Nacalai Tesque, Kyoto, Japan); and Hypercarb porous graphitic carbon $(\mathrm{PGC}), 10 \mathrm{~mm} \times 4.6 \mathrm{~mm}, 3 \mu \mathrm{m}$ (Thermo Fisher Scientific, Waltham, MA, USA). The 2D-GC system consisted of a $50 \%$ phenyl methylpolysiloxane column [low thermal mass (LTM) column module DB-17 ms], $30 \mathrm{~m} \times$ $0.25 \mathrm{~mm}, 0.25-\mu \mathrm{m}$ phase (Agilent Technologies, Folsom, CA, USA), and an LC50 column, $5 \mathrm{~m} \times 0.25 \mathrm{~mm}, 0.10-\mu \mathrm{m}$ phase (J\&K Scientific, Milton, Canada). In the present study, the first dimension of the 2D-GC system was replaced with a shorter and thinner column $(15 \mathrm{~m} \times 0.25 \mathrm{~mm}, 0.15 \mu \mathrm{m}$; LTM DB-17 ms, Agilent Technologies, Folsom, CA, USA). Also, the detection method was modified by our changing the detectors from FIDs to MSDs. This was done by attachment of an additional MSD, a Finnigan TSQ 7000 triple-quadrupole mass spectrometer (Thermo Fisher Scientific, Waltham, MA, USA), onto the 7890A GC/5975C MSD (Agilent Technologies, Palo Alto, CA, USA) as shown in Fig. S1.

\section{Analysis by 2D-LC/2D-GC/MS}

The automated clean-up, separation and detection scheme was illustrated previously [25]. In brief, $50 \mu \mathrm{L}$ of IS-spiked extract was injected and passed through the first column $\left(\mathrm{C}_{18}\right)$ with the mobile phase of methanol $(1 \mathrm{~mL} / \mathrm{min})$. After the polar impurities had been sent to the waste, the flow was reversed to the second column (porous graphitic carbon) for peak focusing and mobile phase exchange. Before elution of the compound corresponding to the peak from the PGC column, the column flow was reversed again, and then directed to the third column (5-pentabromobenzyloxypropyl) with the mobile phase of MTBE (1 mL/min). The aliphatics and small aromatic PAHs (mono-PAHs and di-PAHs) were eluted earlier and sent to the waste. By reversal of the flow again, the PAH fraction was eluted as one backflush peak in the chromatogram and collected in the $500-\mu \mathrm{L}$ injection loop of the programmable temperature vaporizer inlet of the GC/MS system via a transfer line (high-temperature deactivated fused-silica tubing). Then, large-volume injection was performed in solvent vent mode with a lowered flow rate $(0.3 \mathrm{~mL} / \mathrm{min})$ of
MTBE. The oven was kept at $50{ }^{\circ} \mathrm{C}$ until the injection had finished. Then, the sample went through the 2D-GC/MS system with use of heart cutting with the Deans switch valve programme. Details of the GC/MS parameters and valve programme are presented in Tables S2 and S3.

\section{Results and discussion}

\section{GC method translation}

The different parameters used for method translation are compared in Table 1. The initial method translation was done with Agilent GC method translation software (http://www.agilent. c o m / e n - u s / s u p port/gas - chromatograph y/ gcmethodtranslation) in "Translate" mode, and then modified to improve the resolution. Two different methods were investigated in this study. The first method (method 1) used a 20-m-long column as the second dimension to compensate for the decreased column efficiency due to the use of helium as the carrier gas. The other method (method 2) used a shorter first-dimension column $(15 \mathrm{~m})$ with higher phase ratio and with the second-dimension column kept short. The resolution gain from the longer column in method 1 was insufficient to overcome the low resolution from the first column. Method 2, instead, was chosen for further method development because of better separation in the first dimension. The total run time was $105 \mathrm{~min}$, including the $20 \mathrm{~min}$ required for the sample injection and clean-up in the 2D-LC, whereas the previous system required $50 \mathrm{~min}$ as described previously [25]. This can be explained by the combined effect of the use of helium gas and inclusion of the late eluted dibenzopyrenes in this study.

Method 2 had much better peak separation in the first dimension than method 1 , especially for PAHs with $\mathrm{m} / \mathrm{z}$ 216 (1-methylfluoranthene and $11 H$-benzo[a]fluorene) and $m / z 252$ (benzo[b]fluoranthene and benzo[ $k]$ fluoranthene) as shown in Fig. 1. All 53 PAH standards, including seven deuterated ISs, were separated as shown in the GC/MS chromatogram (Fig. 2). Of six unresolved peaks from the previous 2D system (peaks corresponding to phenanthrene- $d_{10}$ /phenanthrene, 9-methylphenanthrene/ 1 -methylphenanthrene, pyrene- $d_{10} /$ pyrene, benzo[c]phenanthrene/benzo[ghi]fluoranthene, 6-methylchrysene/ 2-methylchrysene and benzo $[a]$ pyrene- $d_{12} /$ benzo $[a]$ pyrene) [25], the three PAH/IS pairs and benzo[c]phenanthrene/benzo[ghi]fluoranthene could be easily resolved by the MSDs. The remaining two pairs of PAH isomers (with $\mathrm{m} / \mathrm{z} 192$ and 242) were well separated with the current $2 \mathrm{D}$ system as shown in Fig. 2. Benzo $[b]$ fluoranthene and benzo $[k]$ fluoranthene, however, were partially co-eluted despite the use of the first column with a higher phase ratio. A four times longer column 
Table 1 Comparison of method translation parameters between the previous and current two-dimensional (2D) gas chromatography $(G C)$ systems

\begin{tabular}{|c|c|c|c|c|}
\hline Parameters & Previous $^{\mathrm{a}}$ & Method 1 & Method 2 & Reference $^{b}$ \\
\hline System & 2D-LC/2D-GC/FID & 2D-LC/2D-GC/MS & 2D-LC/2D-GC/MS & $\mathrm{LC}-\mathrm{GC} / \mathrm{MS}$ \\
\hline Column outlet pressure & Atmospheric & Vacuum & Vacuum & Vacuum \\
\hline Carrier gas & Hydrogen & Helium & Helium & Helium \\
\hline Carrier gas flow & $\begin{array}{l}\text { Pressure programme, } \\
20-25 \text { psi }\end{array}$ & Constant flow, $1 \mathrm{~mL} / \mathrm{min}$ & Constant flow, $1 \mathrm{~mL} / \mathrm{min}$ & $\begin{array}{l}\text { Constant flow, } 1 \\
\mathrm{~mL} / \mathrm{min}\end{array}$ \\
\hline Column dimension & $\begin{array}{l}\text { 1st: DB-17 ms, } 30 \mathrm{~m} \\
\quad \times 0.25 \mathrm{~mm}, 0.25 \mu \mathrm{m} \\
\text { 2nd: } \mathrm{LC} 50,5 \mathrm{~m} \times 0.25 \\
\quad \mathrm{~mm}, 0.10 \mu \mathrm{m}\end{array}$ & $\begin{array}{l}\text { 1st: DB-17 ms, } 30 \mathrm{~m} \times \\
0.25 \mathrm{~mm}, 0.25 \mu \mathrm{m} \\
\text { 2nd: LC50, } 20 \mathrm{~m} \times 0.25 \\
\text { mm, } 0.10 \mu \mathrm{m}\end{array}$ & $\begin{array}{l}\text { 1st: DB-17 ms, } 15 \mathrm{~m} \times 0.25 \\
\text { mm, } 0.15 \mu \mathrm{m} \\
\text { 2nd: LC } 50,5 \mathrm{~m} \times 0.25 \mathrm{~mm} \text {, } \\
\quad 0.10 \mu \mathrm{m}\end{array}$ & $\begin{array}{l}\text { 1st: DB-17 ms, } \\
60 \mathrm{~m} \times 0.25 \mathrm{~mm} \text {, } \\
0.15 \mu \mathrm{m}\end{array}$ \\
\hline Phase ratio & 1st: 250,2 nd: 625 & 1st: 250,2 nd: 625 & 1st: 417,2 nd: 625 & 1st: 417 \\
\hline
\end{tabular}

FID flame ionization detection, $L C$ liquid chromatography, LC50 50\% liquid crystal polysiloxane, MS mass spectrometry

${ }^{\text {a }}$ Ahmed et al. [25]

${ }^{\mathrm{b}}$ Sadiktsis et al. [26]

(60 m) was even unable to fully resolve those PAHs [26]. In addition, the attempt to separate them in the second dimension changed the elution order and created another co-elution problem, as shown previously [14]. The second column had better resolution for the four PAH isomers with $\mathrm{m} / \mathrm{z} 216$, but the separation between $11 \mathrm{H}$-benzo[b]fluorene and 2-methylpyrene decreased as seen in Figs. 1 and $2 \mathrm{~b}$. Dibenzopyrenes were analysed on the first column because of peak broadening and the extended run time despite the partial co-elution $(\mathrm{m} / \mathrm{z}, 302$ in Fig. 2a).

\section{Validation: linearity, limit of detection and limit of quantification}

A series of calibration standards were prepared in triplicate and injected into the 2D system. The calibration curves were obtained by our plotting the peak area against the concentration. Calibration curves with seven concentrations were obtained for 43 PAHs, whereas for ten PAHs six levels were used and for seven PAHs five levels were used because of the low detectability at lower concentrations. The partially co-eluted dibenzo $[a, i]$ pyrene and dibenzo $[a, h]$ pyrene were determined together as shown in Table 2. The coefficient of determination $\left(R^{2}\right)$ ranged from 0.987 to 0.998 . The limits of detection and the limits of quantification (LOQs) were determined by injection of standard solutions at signal-to-noise ratios of 3 and 10 respectively.

The LOQs, in general, increased with retention times mainly from the peak broadening. As shown in Fig. S2, the trend was noticeable from indeno[1,2,3-cd]fluoranthene, and the LOQ of the last eluted dibenzo[a,i]pyrene and dibenzo $[a, h]$ pyrene was nearly five times the average value for all PAHs. Apart from the additive effect of both columns on the peak broadening, an increasing temperature gap between the first-column and secondcolumn temperature programmes (final temperature of 320 and $260{ }^{\circ} \mathrm{C}$ respectively) contributed to this trend when the compound from the first column started to be eluted at a temperature exceeding $260{ }^{\circ} \mathrm{C}$, the final

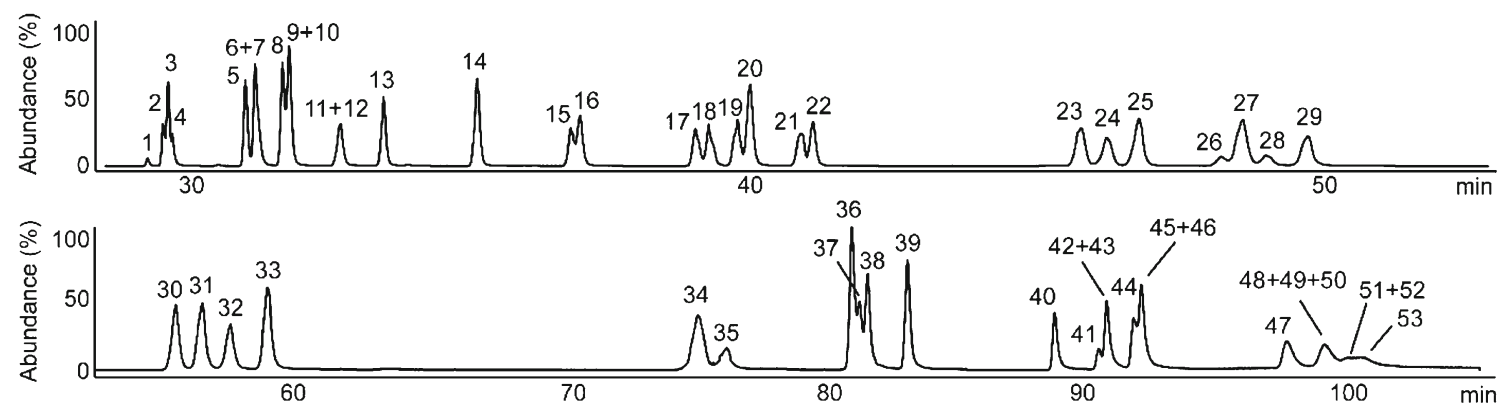

Fig. 1 One-dimensional gas chromatography $(\mathrm{GC}) / \mathrm{mass}$ spectrometry (MS) chromatogram obtained in selected-ion monitoring (SIM) mode with method 2. 1 DBT, 2 Phe- $d_{10}, 3$ Phe, 4 Ant, 5 3-MPhe, 6 2-MPhe, 72-MAnt, 8 9-MPhe, 9 1-MPhe, $104 H$-CPP, 11 9-MAnt, 12 3,6-DMPhe, 133,9-DMPhe, 14 Flu, 15 Pyr- $d_{10}, 16$ Pyr, $171-$ MFlu, $18 \mathrm{~B}[a]$ f, $19 \mathrm{~B}[b]$ f, 20 2-MPyr, 21 4-MPyr, 22 1-MPyr, 23 B[ghi]F, 24 B[c]Phe, 25 B[b]NT,
$26 \mathrm{~B}[a] \mathrm{A}-d_{12}, 27 \mathrm{~B}[a] \mathrm{A}, 28 \mathrm{CPP}, 29 \mathrm{Chr}, 30$ 3-MChr, 31 2-MChr, $326-$ MChr, $331-\mathrm{MChr}, 34 \mathrm{~B}[b] \mathrm{F}, 35 \mathrm{~B}[k] \mathrm{F}, 36 \mathrm{~B}[e] \mathrm{P}, 37 \mathrm{~B}[a] \mathrm{P}-d_{12}, 38 \mathrm{~B}[a] \mathrm{P}$, 39 Per, $40 \mathrm{I}[1,2,3-c d] \mathrm{F}, 41$ unknown, $42 \mathrm{I}[1,2,3-c d] \mathrm{P}, 43 \mathrm{DB}[a, h] \mathrm{A}, 44$ Pic, $45 \mathrm{~B}[g h i] \mathrm{p}-d_{12}, 46 \mathrm{~B}[g h i] \mathrm{p}, 47 \mathrm{DB}[a, l] \mathrm{P}, 48$ Cor- $d_{12}, 49$ Cor, 50 $\mathrm{DB}[a, e] \mathrm{P}, 51 \mathrm{DB}[a, i] \mathrm{P}-d_{14}, 52 \mathrm{DB}[a, i] \mathrm{P}, 53 \mathrm{DB}[a, h] \mathrm{P}$. See Table 2 for an explanation of the abbreviations 

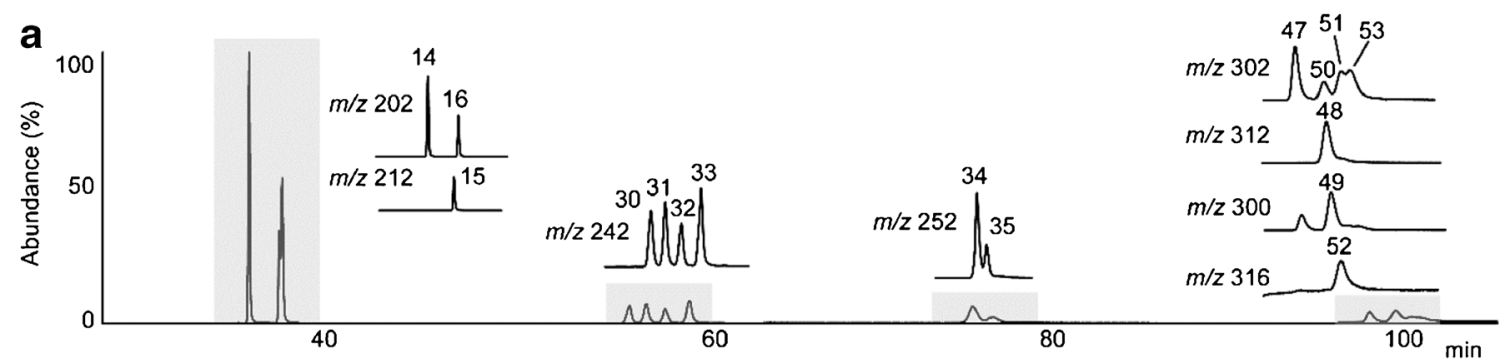

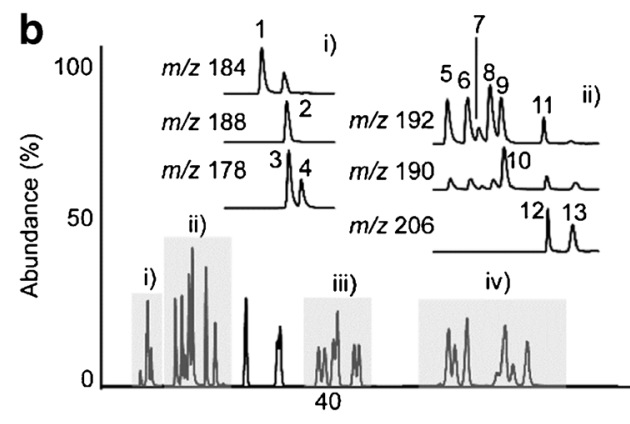

Fig. 2 Two-dimensional (2D) GC/MS chromatogram obtained in SIM mode in the first dimension (a) and second dimension (b) with method 2 showing polycyclic aromatic hydrocarbon (PAH) isomer separation in the enlarged extracted ion chromatograms. 1 DBT, 2 Phe- $d_{10}, 3$ Phe, 4 Ant, 5 3-MPhe, 6 2-MPhe, 7 2-MAnt, 8 9-MPhe, 9 1-MPhe, 10 4H-CPP, 11 9MAnt, 12 3,6-DMPhe, 13 3,9-DMPhe, 14 Flu, 15 Pyr- $d_{10}, 16$ Pyr, 17 1MFlu, 18 B $[a]$ f, 19 B [b]f, 20 2-MPyr, 21 4-MPyr, 22 1-MPyr, 23 B [ghi]F,

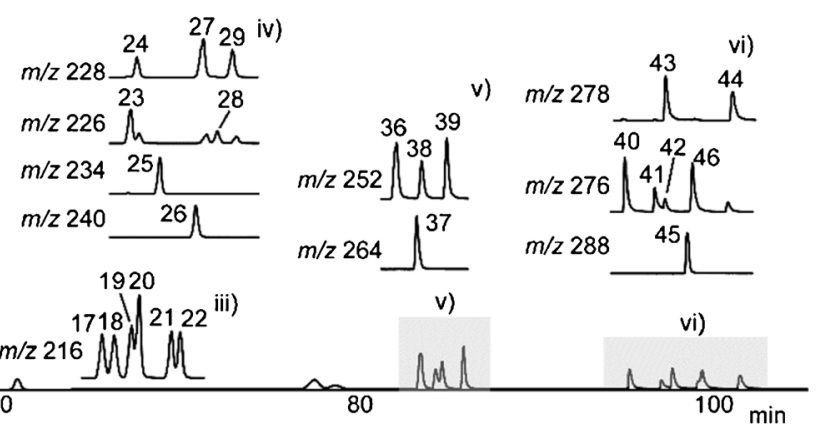

$24 \mathrm{~B}[c] \mathrm{Phe}, 25 \mathrm{~B}[b] \mathrm{NT}, 26 \mathrm{~B}[a] \mathrm{A}-d_{12}, 27 \mathrm{~B}[a] \mathrm{A}, 28 \mathrm{CPP}, 29 \mathrm{Chr}, 303-$ MChr, 31 2-MChr, $326-\mathrm{MChr}, 33$ 1-MChr, $34 \mathrm{~B}[b] \mathrm{F}, 35 \mathrm{~B}[k] \mathrm{F}, 36 \mathrm{~B}[e] \mathrm{P}$, $37 \mathrm{~B}[a] \mathrm{P}-d_{12}, 38 \mathrm{~B}[a] \mathrm{P}, 39 \mathrm{Per}, 40 \mathrm{I}[1,2,3-c d] \mathrm{F}, 41$ unknown, $42 \mathrm{I}[1,2,3-$ $c d] \mathrm{P}, 43 \mathrm{DB}[a, h] \mathrm{A}, 44 \mathrm{Pic}, 45 \mathrm{~B}[g h i] \mathrm{p}-d_{12}, 46 \mathrm{~B}[g h i] \mathrm{p}, 47 \mathrm{DB}[a, l] \mathrm{P}, 48$ Cor- $d_{12}, 49$ Cor, $50 \mathrm{DB}[a, e] \mathrm{P}, 51 \mathrm{DB}[a, i] \mathrm{P}-d_{14}, 52 \mathrm{DB}[a, i] \mathrm{P}, 53$ $\mathrm{DB}[a, h] \mathrm{P}$. See Table 2 for an explanation of the abbreviations

preparation and examine the long-term storage stability of the crude extract. A representative chromatogram for SRM 1649a is presented in Fig. 3. The problematic PAH isomers with $\mathrm{m} / \mathrm{z} 226$ (cyclopenta[ $\mathrm{cd}]$ pyrene), $\mathrm{m} / \mathrm{z} 228$ (benz $[a]$ anthracene, chrysene and triphenylene) and $\mathrm{m} / \mathrm{z}$ 252 (benzo $[b]$ fluoranthene, benzo $[k]$ fluoranthene, benzo[j]fluoranthene and benzo $[a]$ fluoranthene) could not be separated, especially triphenylene and chrysene because they are normally co-eluted on the DB17-ms column [14].

\section{SRM 1649a (urban dust) and SRM 1975 (diesel particulate extract)}

The PAH concentrations of SRM 1649a (urban dust) from this study were compared with those from the previous study together with NIST certified values as seen in Fig. 4. Most of the concentrations were within $20 \%$ or slightly higher (21\% for picene) of the previous values except for anthracene, perylene and indeno[1,2,3-cd]pyrene. In the case of anthracene, the value was in good agreement with that obtained with the previous system but $64 \%$ higher than that from the NIST. The overestimation, however, was largely because of different extraction methods: the present study used pressurized liquid extraction and the NIST used classic Soxhlet extraction [33]. The difference was $-28 \%$ when compared with the reference
Two SRMs, urban dust (SRM 1649a) and diesel particulate extract (SRM 1975), were used to validate the method developed. Furthermore, the 2D system was applied to a real sample, wood smoke particulates. All the samples were prepared at the same time as those used in the previous study [25] to minimize the variation in the sample 
Table 2 Summary of the linear range, correlation coefficient, limit of detection $(L O D)$ and limit of quantification $(L O Q)$

\begin{tabular}{|c|c|c|c|c|c|c|}
\hline PAH & Abbreviation & $m / z$ & Linear range $(\mathrm{pg})$ & $R^{2}$ & LOD (pg) & LOQ (pg) \\
\hline Dibenzothiophene & DBT & 184 & $78.3-7830$ & 0.992 & 19.6 & 78.3 \\
\hline Phenanthrene & Phe & 178 & $227-22,700$ & 0.993 & 56.8 & 227 \\
\hline Anthracene & Ant & 178 & $79.0-7900$ & 0.994 & 19.7 & 79.0 \\
\hline 3-Methylphenanthrene & 3-MPhe & 192 & $218-21,800$ & 0.991 & 54.5 & 218 \\
\hline 2-Methylphenanthrene & 2-MPhe & 192 & $257-25,700$ & 0.993 & 64.4 & 257 \\
\hline 2-Methylanthracene & 2-MAnt & 192 & $81.2-8120$ & 0.991 & 20.3 & 81.2 \\
\hline 9-Methylphenanthrene & 9-MPhe & 192 & $282-28,200$ & 0.997 & 70.6 & 282 \\
\hline 1-Methylphenanthrene & 1-MPhe & 192 & $202-20,200$ & 0.994 & 50.5 & 202 \\
\hline 4H-Cyclopenta[ $[$ def $]$ phenanthrene & 4H-CPP & 190 & $148-14,800$ & 0.994 & 37.1 & 148 \\
\hline 3,6-Dimethylphenanthrene & 3,6-DMPhe & 206 & $134-13,400$ & 0.996 & 33.5 & 134 \\
\hline 9-Methylanthracene & 9-MAnt & 192 & $148-14,800$ & 0.990 & 37.0 & 148 \\
\hline 3,9-Dimethylphenanthrene & 3,9-DMPhe & 206 & $192-19,200$ & 0.996 & 48.0 & 192 \\
\hline Fluoranthene & Flu & 202 & $862-27,600$ & 0.997 & 2.15 & 8.62 \\
\hline Pyrene & Pyr & 202 & $453-14500$ & 0.996 & 1.13 & 4.53 \\
\hline 1-Methylfluoranthene & 1-MFlu & 216 & $185-18,500$ & 0.995 & 46.2 & 185 \\
\hline $11 H$-Benzo $[a]$ fluorene & $\mathrm{B}[a] \mathrm{f}$ & 216 & $246-24,600$ & 0.993 & 61.6 & 246 \\
\hline $11 H$-Benzo $[b]$ fluorene & $\mathrm{B}[b] \mathrm{f}$ & 216 & $277-27,700$ & 0.994 & 69.4 & 277 \\
\hline 2-Methylpyrene & 2-MPyr & 216 & $261-26,100$ & 0.997 & 32.6 & 65.2 \\
\hline 4-Methylpyrene & 4-MPyr & 216 & $145-14,500$ & 0.994 & 36.3 & 145 \\
\hline 1-Methylpyrene & 1-MPyr & 216 & $170-17,000$ & 0.995 & 42.6 & 170 \\
\hline Benzo $[c]$ phenanthrene ${ }^{a}$ & $\mathrm{~B}[c] \mathrm{Phe}$ & 228 & $316-12,600$ & 0.996 & 126 & 316 \\
\hline Benzo $[g h i]$ fluoranthene & $\mathrm{B}[g h i] \mathrm{F}$ & 226 & $184-18,400$ & 0.997 & 45.9 & 184 \\
\hline Benzo $[b]$ naphtho $[1,2-d]$ thiophene & $\mathrm{B}[b] \mathrm{NT}$ & 234 & $127-12,700$ & 0.998 & 31.7 & 127 \\
\hline Benz $[a]$ anthracene & $\mathrm{B}[a] \mathrm{A}$ & 228 & $273-27,300$ & 0.995 & 68.3 & 273 \\
\hline Cyclopenta $[c d]$ pyrene $^{\mathrm{a}}$ & СPP & 226 & $380-15,200$ & 0.992 & 152 & 380 \\
\hline Chrysene $^{\mathrm{a}}$ & $\mathrm{Chr}$ & 228 & $443-17,700$ & 0.995 & 177 & 443 \\
\hline 3-Methylchrysene & 3-MChr & 242 & $500-16,000$ & 0.997 & 20.0 & 160 \\
\hline 2-Methylchrysene & 2-MChr & 242 & $595-19,000$ & 0.997 & 47.6 & 190 \\
\hline 6-Methylchrysene & 6-MChr & 242 & $432-13,800$ & 0.996 & 34.6 & 138 \\
\hline 1-Methylchrysene & 1-MChr & 242 & $664-21,200$ & 0.998 & 53.1 & 212 \\
\hline Benzo $[b]$ fluoranthene & $\mathrm{B}[b] \mathrm{F}$ & 252 & $804-25,700$ & 0.994 & 257 & 644 \\
\hline Benzo $[k]$ fluoranthene & $\mathrm{B}[k] \mathrm{F}$ & 252 & $366-11,700$ & 0.996 & 117 & 292 \\
\hline Benzo[e]pyrene & $\mathrm{B}[e] \mathrm{P}$ & 252 & $275-27,500$ & 0.991 & 68.7 & 275 \\
\hline $\operatorname{Benzo}[a]$ pyrene $^{\mathrm{a}}$ & $\mathrm{B}[a] \mathrm{P}$ & 252 & $523-20,900$ & 0.995 & 209 & 523 \\
\hline Perylene $^{\mathrm{a}}$ & Per & 252 & $680-27,200$ & 0.996 & 272 & 680 \\
\hline Indeno $[1,2,3-c d]$ fluoranthene ${ }^{\mathrm{b}}$ & $\mathrm{I}[1,2,3-c d] \mathrm{F}$ & 276 & $1180-23,600$ & 0.990 & 591 & 1180 \\
\hline Indeno $[1,2,3-c d]$ pyrene $^{\mathrm{a}}$ & $\mathrm{I}[1,2,3-c d] \mathrm{P}$ & 276 & $786-12,600$ & 0.987 & 314 & 786 \\
\hline Dibenz $[a, h]$ anthracene $^{\mathrm{b}}$ & $\mathrm{DB}[a, h] \mathrm{A}$ & 278 & $1280-25,600$ & 0.989 & 640 & 1280 \\
\hline Benzo $[g h i]$ perylrene ${ }^{\mathrm{b}}$ & $\mathrm{B}[g h i] \mathrm{p}$ & 276 & $1050-21,000$ & 0.990 & 526 & 1050 \\
\hline Picene $^{\mathrm{a}}$ & Pic & 278 & $1540-24,700$ & 0.989 & 617 & 1230 \\
\hline $\operatorname{Dibenzo}[a, l]$ pyrene $^{\mathrm{a}}$ & $\mathrm{DB}[a, l] \mathrm{P}$ & 302 & $1500-23,900$ & 0.997 & 748 & 1500 \\
\hline Dibenzo $[a, e]$ pyrene ${ }^{\mathrm{b}}$ & $\mathrm{DB}[a, e] \mathrm{P}$ & 302 & $1420-11,300$ & 0.996 & 354 & 1420 \\
\hline Coronene $^{\mathrm{a}}$ & Cor & 300 & $1840-29,400$ & 0.995 & 920 & 1840 \\
\hline Dibenzo $[a, i]$ pyrene + dibenzo $[a, h]$ pyrene $^{\mathrm{b}}$ & $\mathrm{DB}[a, i] \mathrm{P}+\mathrm{DB}[a, h] \mathrm{P}$ & 302 & $3490-41,900$ & 0.988 & 1750 & 3490 \\
\hline \multicolumn{7}{|l|}{ Internal standards } \\
\hline Phenanthrene- $d_{10}$ & Phe- $d_{10}$ & 188 & $213-21,300$ & 0.990 & 53.3 & 213 \\
\hline Pyrene- $d_{10}$ & Pyr-d $d_{10}$ & 212 & $432-13,800$ & 0.998 & 8.65 & 34.6 \\
\hline Benz $[a]$ anthracene- $d_{12}{ }^{\mathrm{b}}$ & $\mathrm{B}[a] \mathrm{A}-d_{12}$ & 240 & $518-10,400$ & 0.992 & 104 & 518 \\
\hline Benzo $[a]$ pyrene- $d_{12}{ }^{\mathrm{a}}$ & $\mathrm{B}[a] \mathrm{P}-d_{12}$ & 264 & $534-21,400$ & 0.994 & 214 & 534 \\
\hline
\end{tabular}


Table 2 (continued)

\begin{tabular}{|c|c|c|c|c|c|c|}
\hline PAH & Abbreviation & $m / z$ & Linear range $(\mathrm{pg})$ & $R^{2}$ & LOD (pg) & LOQ (pg) \\
\hline Benzo $[g h i]$ perylrene- $d_{12}{ }^{\mathrm{b}}$ & $\mathrm{B}[g h i] \mathrm{p}-d_{12}$ & 288 & $521-10,400$ & 0.993 & 261 & 521 \\
\hline Coronene- $d_{12}$ & Cor- $d_{12}$ & 300 & $654-10,500$ & 0.997 & 262 & 523 \\
\hline
\end{tabular}

PAH polycyclic aromatic hydrocarbon

${ }^{a}$ Based on the six-level calibration curve

${ }^{\mathrm{b}}$ Based on the five-level calibration curve

value determined with pressurized liquid extraction at $200{ }^{\circ} \mathrm{C}$, strengthening the difference from the applied extraction method [34]. Although the lower chemical stability of anthracene mostly contributed to the underestimation [35], the problem of the abnormally higher value for perylene obtained with the previous system due to interference could be solved by the introduction of the MSD in the present study. The concentration of indeno[1,2,3-cd]pyrene determined in this study correlated well with the concentrations obtained with the previous $2 \mathrm{D}$ system and from the NIST, but not with the concentration obtained with the LC-GC/MS method [25].

The present 2D system was also validated for diesel particulate extract (SRM 1975). As seen in Fig. 5, the PAH concentrations determined were within $20 \%$ of the literature values, and only two values were just above $20 \%$ when compared with those obtained by LC-GC/MS $[25,36]$. In detail, the concentrations of fluoranthene and chrysene were within $23 \%$ and $-21 \%$ of the literature values, among which the slight underestimation of chrysene was owing to better separation between triphenylene and chrysene. However, their concentrations were in good agreement with those obtained with the previous $2 \mathrm{D}$ system and from the NIST.

\section{Wood smoke particulate}

As shown in Fig. 6, the PAH concentrations in the wood smoke particulate sample were compared with those obtained with the previous system and the LC-GC/MS method $[25,27]$. The concentrations were generally within $20 \%$ of the previous values, but the concentrations for 2 methylpyrene and indeno[ $[1,2,3-c d]$ pyrene were within $26 \%$. The determined concentrations of 1-methylchrysene, perylene and coronene were lower than those obtained with the previous $2 \mathrm{D}$ system, which was mainly caused by the difference in the detectors, indicating better selectivity of the MSD compared with the FID. Underestimation

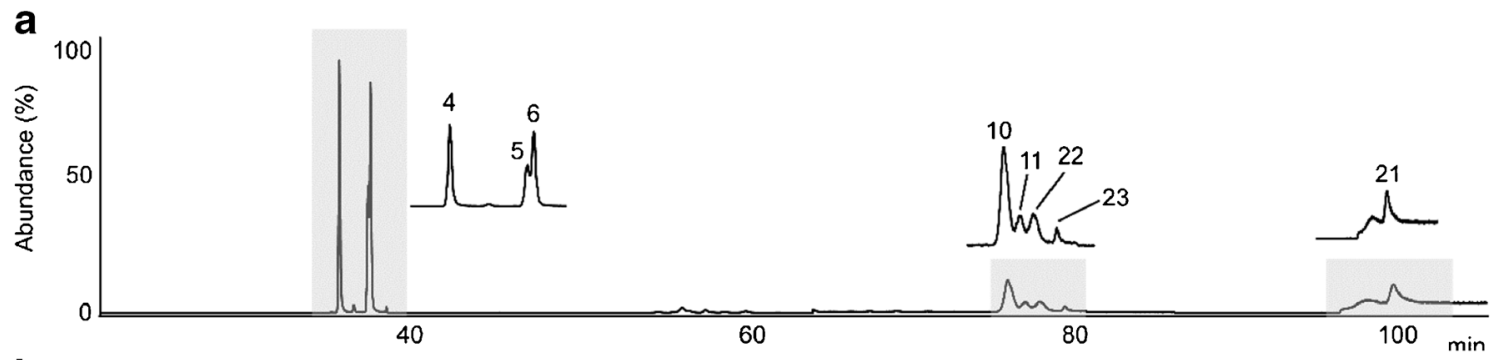

b

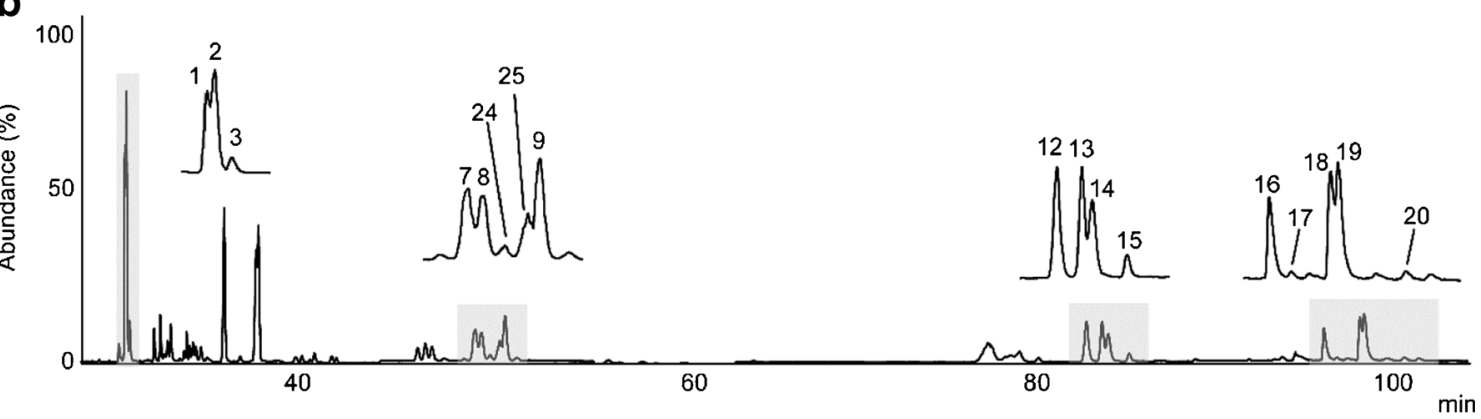

Fig. 3 Two-dimensional GC/MS chromatogram obtained in SIM mode in the first dimension (a) and second dimension (b) obtained from urban dust (SRM 1649a). 1 Phe- $d_{10}, 2$ Phe, 3 Ant, 4 Flu, 5 Pyr- $d_{10}$, 6 Pyr, 7 $\mathrm{B}[a] \mathrm{A}-d_{12}, 8 \mathrm{~B}[a] \mathrm{A}, 9 \mathrm{Chr}, 10 \mathrm{~B}[b] \mathrm{F}, 11 \mathrm{~B}[k] \mathrm{F}, 12 \mathrm{~B}[e] \mathrm{P}, 13 \mathrm{~B}[a] \mathrm{P}-d_{12}$,
$14 \mathrm{~B}[a] \mathrm{P}, 15$ Per, $16 \mathrm{I}[1,2,3-c d] \mathrm{P}, 17 \mathrm{DB}[a, h] \mathrm{A}, 18 \mathrm{~B}[g h i] \mathrm{p}-d_{12}, 19$ $\mathrm{B}[g h i] \mathrm{p}, 20 \mathrm{Pic}, 21 \mathrm{DB}[a, e] \mathrm{P}, 22$ benzo[j]fluoranthene, 23 benzo[a]fluoranthene, 24 CPP, 25 triphenylene. See Table 2 for an explanation of the abbreviations 


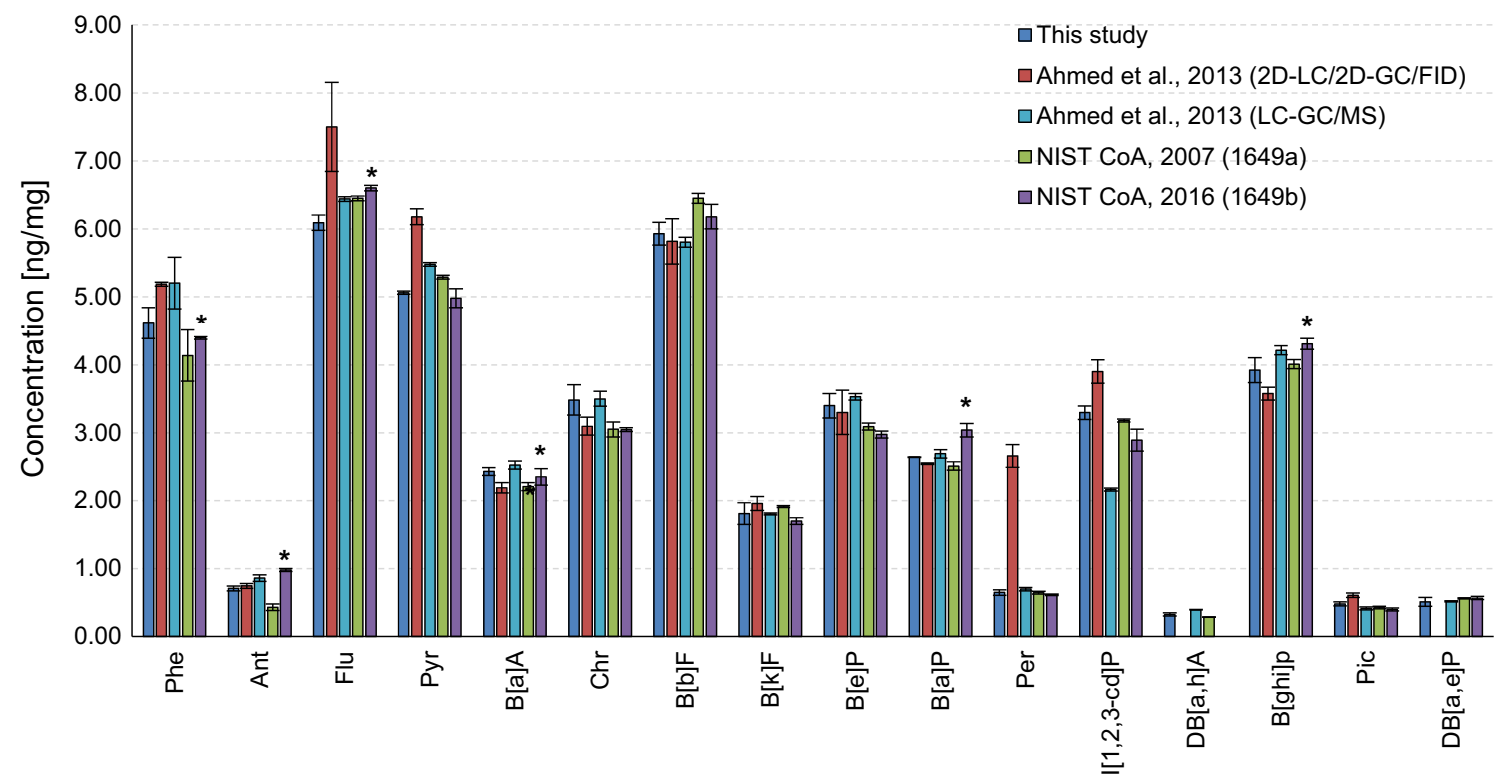

Fig. 4 Comparison of results for PAH determination in SRM 1649a (urban dust) from the present study and the previous data determined by $2 \mathrm{D}$ liquid chromatography (LC)/2D-GC/flame ionization detection (FID) [25] and LC-GC/MS followed by solid-phase extraction (SPE)

of another PAH, cyclopenta $[c d]$ pyrene, when compared with the concentration determined by $\mathrm{LC}-\mathrm{GC} / \mathrm{MS}$, on the other hand, was the result of improved separation of the 2D system regardless of the detectors. The overestimation of 3,6-dimethylphenanthrene with both 2D systems, however, was due to lower column efficiency than in the $\mathrm{LC}-\mathrm{GC} /$ MS method, where a 60-m column was used. The concentration of indeno[1,2,3-cd]fluoranthene was abnormally
[25] and National Institute of Standards and Technology (NIST) certified values $[33,34]$. See Table 2 for an explanation of the abbreviations. Asterisks reference mass fraction values based on pressurized fluid extraction at $200{ }^{\circ} \mathrm{C}$

high (approximately 300\%) in the present study compared with previous studies. The sample matrix under long-term storage was thought to affect the stability of the fluoranthene-based compound, implying the determination of indeno[ $[1,2,3-c d]$ fluoranthene should be done with care when it is stored for a long period.

In summary, the present $2 \mathrm{D}$ system was considered to be valid for PAH determination from three different matrices:

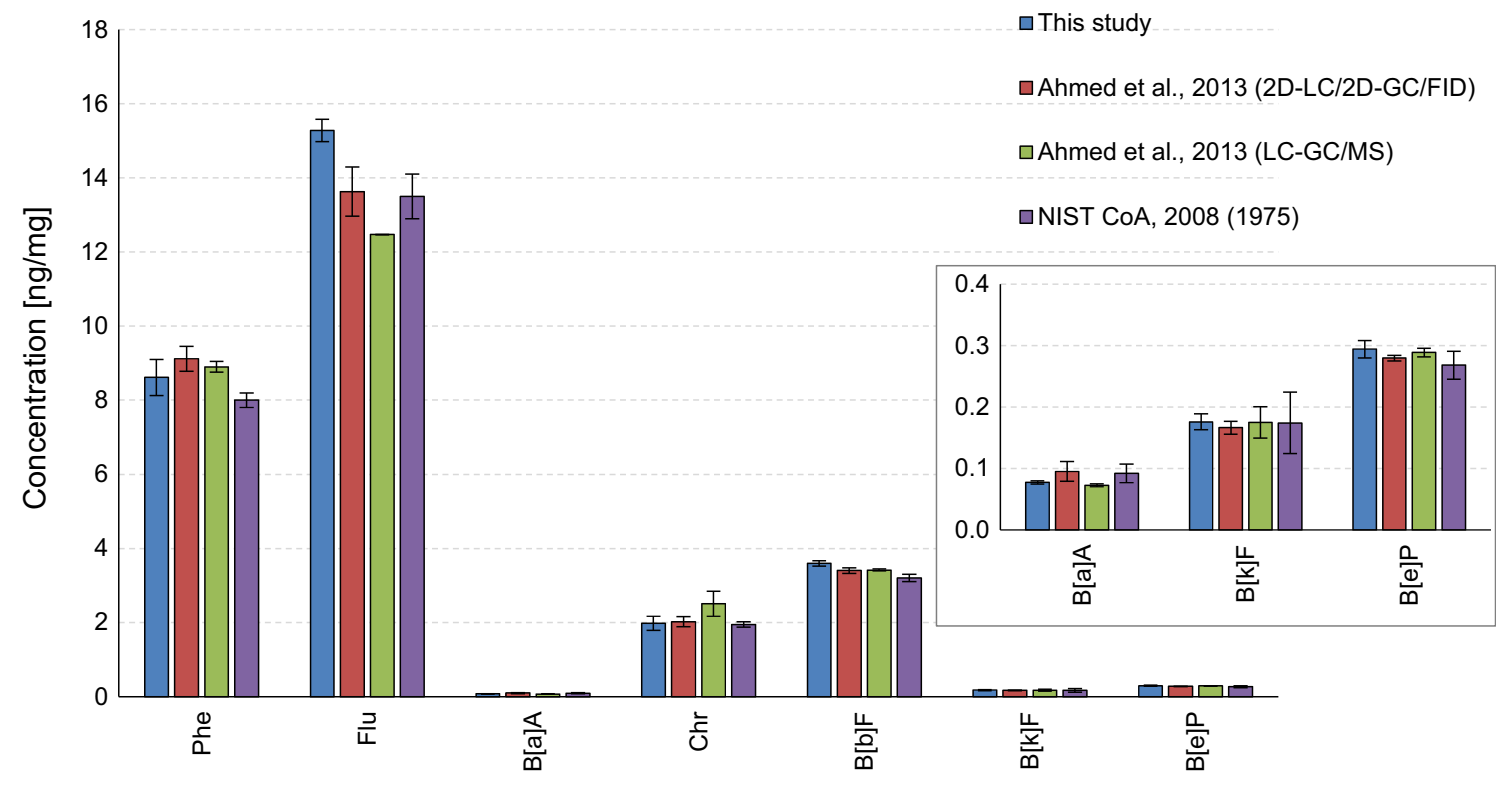

Fig. 5 Comparison of results for PAH determination in SRM 1975 (diesel particulate extract) from the present study and previous data determined by 2D-LC/2D-GC/FID [25] and LC-GC/MS followed by SPE [25] and NIST certified values [36]. See Table 2 for an explanation of the abbreviations 

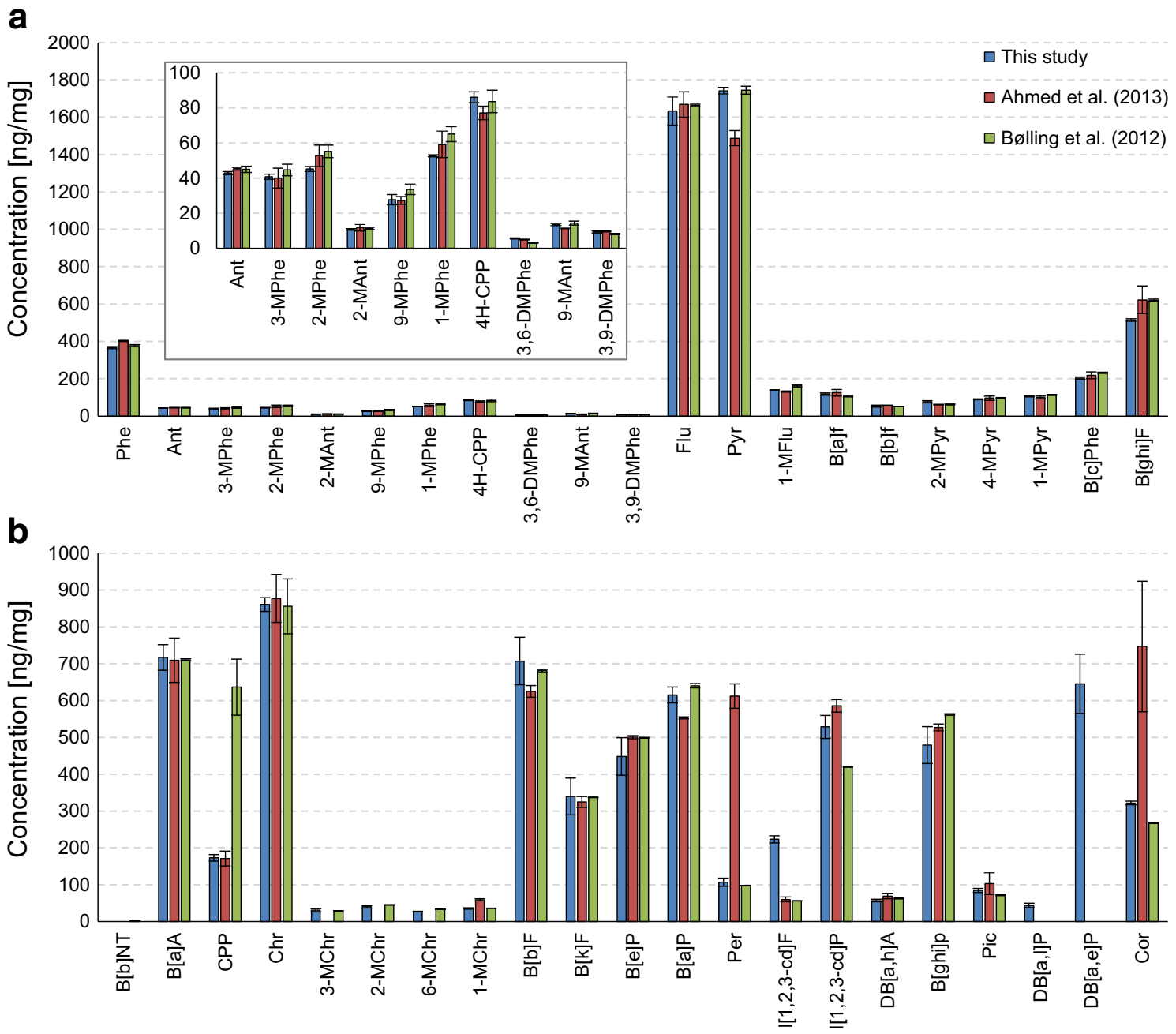

Fig. 6 Comparison of results for the PAH determination in the wood smoke particulate sample from the present study and the previous data determined by 2D-LC/2D-GC/FID [25] and LC-GC/MS followed by

urban dust, diesel particulate matter extract and wood smoke particulates. The determined concentrations, in general, agreed better with those obtained with the previous 2D system and from the NIST than those obtained by LCGC/MS. Additionally, the long-term storage of the crude extract was mostly acceptable when compared with the previous results. However, anthracene from urban dust (SRM 1649a) and indeno[1,2,3-cd]fluoranthene from the wood smoke particulate sample were more susceptible to the storage condition and matrix type. All the PAH concentrations are listed together with the previously reported data in Tables S4, S5 and S6.

\section{Conclusion}

We developed an automated 2D-LC/2D-GC system with two MSDs based on the previous system with two FIDs to improve the detectability and selectivity of PAHs in
SPE [27] from phenanthrene (Phe) to benzo[ghi]fluoranthene $(B[g h i] F)$ (a) and from benzo $[b]$ naphtho[1,2- $d]$ thiophene $(B[b] N T)$ to coronene (Cor) (b). See Table 2 for an explanation of the abbreviations

complex environmental matrices. The detector change involved the use of helium as the carrier gas, resulting in decreased column efficiency. Hence the method translation involved not only the carrier gas but also the column dimension to improve the separation. Two SRMs from the NIST, urban dust (SRM 1649a) and diesel particulate extract (SRM 1975), and a wood smoke particulate sample were used to validate the system developed. The results showed good correlation with those reported previously, especially greater agreement with the values obtained with the previous $2 \mathrm{D}$ system and from the NIST than those obtained by $1 \mathrm{D}-\mathrm{LC}-\mathrm{GC} / \mathrm{MS}$. The crude extracts originally prepared in the previous study and stored at $-20{ }^{\circ} \mathrm{C}$ until analysis showed acceptable stability except for anthracene and indeno[1,2,3-cd]fluoranthene, possibly due to their chemical stability or the influence of the matrix during storage.

The 2D system developed enhanced the validity of the previous system and has the potential to be further developed 
by introduction of novel stationary phases in the second dimension.

Acknowledgements The authors are grateful to Michael Strandell and Ioannis Athanassiadis for technical support with regard to operation of the TSQ 7000 triple-quadrupole mass spectrometer. This work was supported by Stockholm University.

\section{Compliance with ethical standards}

Funding This study was funded by Stockholm University.

Conflict of interest The authors declare that they have no conflict of interest.

Open Access This article is distributed under the terms of the Creative Commons Attribution 4.0 International License (http:// creativecommons.org/licenses/by/4.0/), which permits unrestricted use, distribution, and reproduction in any medium, provided you give appropriate credit to the original author(s) and the source, provide a link to the Creative Commons license, and indicate if changes were made.

\section{References}

1. Mateus EP, da Silva MDRG, Ribeiro AB, Marriott P. Multidimensional chromatographic techniques for monitoring and characterization of environmental samples. In: Ribeiro AB, Mateus EP, Couto N, editors. Electrokinetics across disciplines and continents: new strategies for sustainable development. Cham: Springer; 2016. 439-53.

2. Bertsch W. Two-dimensional gas chromatography. Concepts, instrumentation, and applications - part 1: fundamentals, conventional two-dimensional gas chromatography, selected applications. J High Resolut Chromatogr. 1999;22:647-65.

3. Simmons MC, Snyder LR. Two-stage gas-liquid chromatography. Anal Chem. 1958;30:32-5.

4. Liu Z, Phillips JB. Comprehensive two-dimensional gas-chromatography using an on-column thermal modulator interface. J Chromatogr Sci. 1991;29:227-31.

5. Giddings JC. Concepts and comparisons in multidimensional separation. J High Resolut Chromatogr. 1987;10:319-23.

6. Tranchida PQ, Sciarrone D, Dugo P, Mondello L. Heart-cutting multidimensional gas chromatography: A review of recent evolution, applications, and future prospects. Anal Chim Acta. 2012;716: 66-75.

7. Chin ST, Marriott PJ. Multidimensional gas chromatography beyond simple volatiles separation. Chem Commun. 2014;50:881933.

8. Andersson JT. PAH or PAC, that is the question. Polycycl Aromat Compd. 2009;29:1-2.

9. Lewtas J. Air pollution combustion emissions: characterization of causative agents and mechanisms associated with cancer, reproductive, and cardiovascular effects. Mutat Res. 2007;636:95-133.

10. Boström CE, Gerde P, Hanberg A, Jernström B, Johansson C, Kyrklund T, et al. Cancer risk assessment, indicators, and guidelines for polycyclic aromatic hydrocarbons in the ambient air. Environ Health Persp. 2002;110 Suppl 3:451-88.

11. IARC. Working Group on the Evaluation of Carcinogenic Risk to Humans. Some non-heterocyclic polycyclic aromatic hydrocarbons and some related exposures. IARC monographs on the evaluation of carcinogenic risks to humans. Vol. 92. Lyon: International Agency for Research on Cancer; 2010.
12. IARC. Working Group on the Evaluation of Carcinogenic Risk to Humans. Chemical agents and related occupations: a review of human carcinogens. IARC monographs on the evaluation of carcinogenic risks to humans. Vol. 100F. Lyon: International Agency for Research on Cancer; 2012.

13. IARC. Working Group on the Evaluation of Carcinogenic Risk to Humans. Outdoor air pollution. IARC monographs on the evaluation of carcinogenic risks to humans. Vol. 109. Lyon: International Agency for Research on Cancer; 2016.

14. Poster DL, Schantz MM, Sander LC, Wise SA. Analysis of polycyclic aromatic hydrocarbons (PAHs) in environmental samples: a critical review of gas chromatographic (GC) methods. Anal Bioanal Chem. 2006;386:859-81.

15. Wise SA, Sander LC, Schantz MM. Analytical methods for determination of polycyclic aromatic hydrocarbons (PAHs) - a historical perspective on the 16 U.S. EPA priority pollutant PAHs. Polycycl Aromat Compd. 2015;35:187-247.

16. Wise SA, Chesler SN, Hertz HS, Hilpert LR, May WE. Chemically-bonded aminosilane stationary phase for the highperformance liquid chromatographic separation of polynuclear aromatic compounds. Anal Chem. 1977;49:2306-10.

17. Sonnefeld WJ, Zoller WH, May WE, Wise SA. Online multidimensional liquid chromatographic determination of polynuclear aromatic hydrocarbons in complex samples. Anal Chem. 1982;54: 723-7.

18. Venkatramani CJ, Xu J, Phillips JB. Separation orthogonality in temperature-programmed comprehensive two-dimensional gas chromatography. Anal Chem. 1996;68:1486-92.

19. Marriott PJ, Haglund P, Ong RCY. A review of environmental toxicant analysis by using multidimensional gas chromatography and comprehensive GC. Clin Chim Acta. 2003;328:1-19.

20. Adahchour M, Brinkman UAT. Multidimensional and comprehensive two-dimensional gas chromatography. In: Dettmer-Wilde K, Engewald W, editors. Practical gas chromatography: a comprehensive reference. Berlin: Springer; 2014. p. 461-502.

21. Wise SA, Sander LC, May WE. Determination of polycyclic aromatic hydrocarbons by liquid chromatography. J Chromatogr A. 1993;642:329-49.

22. Wise SA, Sander LC, Chang HCK, Markides KE, Lee ML. Shape selectivity in liquid and gas chromatography: polymeric octadecylsilane $\left(\mathrm{C}_{18}\right)$ and liquid crystalline stationary phases. Chromatographia. 1988;25:473-9.

23. Manzano C, Hoh E, Simonich SLM. Improved separation of complex polycyclic aromatic hydrocarbon mixtures using novel column combinations in GC $\times$ GC/ToF-MS. Environ Sci Technol. 2012;46: 7677-84.

24. Manzano C, Hoh E, Simonich SLM. Quantification of complex polycyclic aromatic hydrocarbon mixtures in standard reference materials using comprehensive two-dimensional gas chromatography with time-of-flight mass spectrometry. J Chromatogr A. 2013;1307:172-9.

25. Ahmed TM, Lim H, Bergvall C, Westerholm R. Automated cleanup, separation and detection of polycyclic aromatic hydrocarbons in particulate matter extracts from urban dust and diesel standard reference materials using a $2 \mathrm{D}-\mathrm{LC} / 2 \mathrm{D}-\mathrm{GC}$ system. Anal Bioanal Chem. 2013;405:8215-22.

26. Sadiktsis I, Koegler JH, Benham T, Bergvall C, Westerholm R. Particulate associated polycyclic aromatic hydrocarbon exhaust emissions from a portable power generator fueled with three different fuels - a comparison between petroleum diesel and two biodiesels. Fuel. 2014;115:573-80.

27. Bølling AK, Totlandsdal AI, Sallsten G, Braun A, Westerholm $\mathrm{R}$, Bergvall C, et al. Wood smoke particles from different combustion phases induce similar pro-inflammatory effects in a coculture of monocyte and pneumocyte cell lines. Part Fibre Toxicol. 2012;9:45. 
28. Masala S, Ahmed T, Bergvall C, Westerholm R. Improved efficiency of extraction of polycyclic aromatic hydrocarbons (PAHs) from the National Institute of Standards and Technology (NIST) Standard Reference Material diesel particulate matter (SRM 2975) using accelerated solvent extraction. Anal Bioanal Chem. 2011;401:3305-15.

29. Wilson WB, Sander LC, Oña-Rualesa JO, Mössner SG, Sidisky LM, Lee ML, et al. Retention behavior of isomeric polycyclic aromatic sulfur heterocycles in gas chromatography on stationary phases of different selectivity. J Chromatogr A. 2017;1485:120-30.

30. Anderson JL, Armstrong DW. Immobilized ionic liquids as highselectivity/high-temperature/high-stability gas chromatography stationary phases. Anal Chem. 2005;77:6453-62.

31. Dutriez T, Borras J, Courtiade M, Thiébaut D, Dulot H, Bertoncini $\mathrm{F}$, et al. Challenge in the speciation of nitrogen-containing compounds in heavy petroleum fractions by high temperature comprehensive two-dimensional gas chromatography. J Chromatogr A. 2011;1218:3190-9.

32. Antle P, Zeigler C, Robbat Jr A. Retention behavior of alkylated polycyclic aromatic sulfur heterocycles on immobilized ionic liquid stationary phases. J Chromatogr A. 2014;1361:255-64.

33. National Institute of Standards and Technology. Certificate of analysis: Standard Reference Material 1649a urban dust. Gaithersburg: National Institute of Standards and Technology; 2007.

34. National Institute of Standards and Technology. Certificate of analysis: Standard Reference Material 1649b urban dust. Gaithersburg: National Institute of Standards and Technology; 2016.

35. Lee ML, Novotny MV, Bartle KD. Analytical chemistry of polycyclic aromatic compounds. New York: Academic; 1981.

36. National Institute of Standards and Technology. Certificate of analysis: Standard Reference Material 1975 diesel particulate extract. Gaithersburg: National Institute of Standards and Technology; 2008.

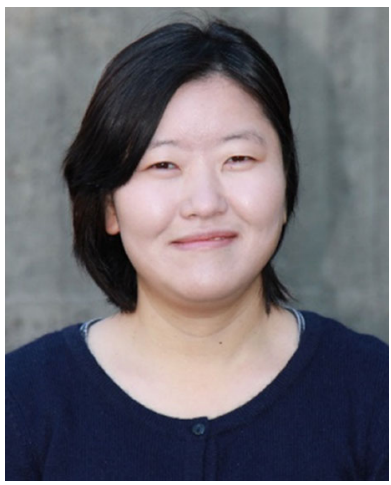

Hwanmi Lim is a postgraduate student in the Department of Environmental Science and Analytical Chemistry, Stockholm University, and has worked on method development for polycyclic aromatic hydrocarbon analysis using multidimensional chromatographic and mass spectrometric techniques.

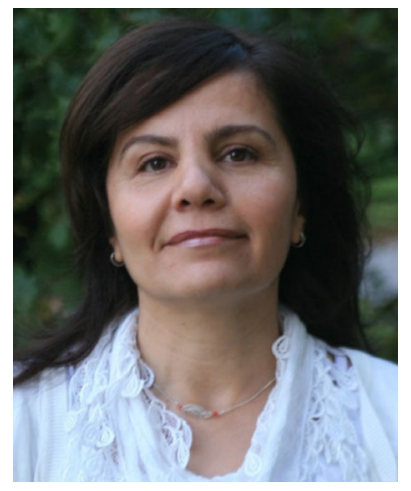

Trifa M. Ahmed has a $\mathrm{PhD}$ degree in analytical chemistry from Stockholm University. She has been working on the development and validation of analytical chemical methods for trace analysis of carcinogenic organic compounds using multidimensional chromatographic methods consisting of liquid chromatography coupled with gas chromatography/mass spectrometry. She is a chemist at the Swedish National Food Agency.

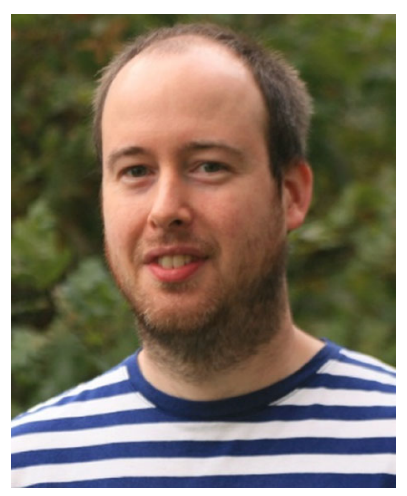

Christoffer Bergvall is an analytical chemist. He studied and obtained his $\mathrm{PhD}$ degree in the Department of Analytical Chemistry at Stockholm University. His research interest is the development and validation of methods for the determination of polycyclic aromatic compounds. Special focus has been on the use of highly automated and multidimensional methods consisting of liquid chromatography coupled with gas chromatography/ mass spectrometry.

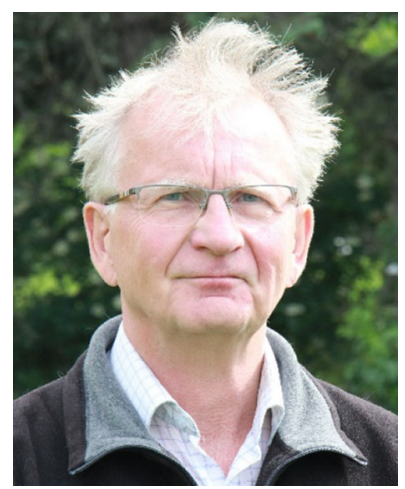

Roger Westerholm is Professor of Analytical Chemistry in the Department of Environmental Science and Analytical Chemistry, Stockholm University. His research covers a broad spectrum of analytical methods, from sampling techniques to online analytical techniques of liquid/gas chromatography coupled with mass spectrometry. The compounds of interest are polycyclic aromatic compounds in general. 